\title{
Open-label Compassionate Use One Year-treatment with Pirfenidone to Patients with Chronic Pulmonary Fibrosis
}

\author{
Sonoko Nagai, Kunio Hamada, Michio Shigematsu, Masayosi TaniYama*, \\ Shitotomo YAMAUCHI* and Takateru IzUMI
}

\begin{abstract}
Objective We attempted to evaluate the feasibility (therapeutic efficacy, tolerance, and clinical courses after treatment) of pirfenidone, an anti-fibrotic drug for patients with chronic pulmonary fibroses such as idiopathic pulmonary fibrosis (IPF).

Methods Open-label one-year treatment for compassionate-use. Patients or Materials: Oral pirfenidone $(40 \mathrm{mg} /$ kg body weight) was administered to 8 patients with advanced IPF and 2 with interstitial pneumonia associated with diffuse systemic sclerosis. The plasma concentration of the drug was serially followed. Radiographic scores, pulmonary functions, and arterial blood oxygen pressure were compared at three time points: at one-year before treatment, at the time of entry, and at one-year after entry.

Results While pirfenidone did not show a definite therapeutic effect on overall survival (2 years after entry). During one-year treatment, there was no significant deterioration in terms of chest radiographic scores and arterial oxygen pressure, and the drug was well tolerated with minimal adverse effects within the ranges observed in this study, plasma pirfenidone concentrations did not seem to relate to the appearance of adverse effects or differences in therapeutic effects.

Conclusion The feasibility of Pirfenidone as a therapeutic drug was confirmed for patients with advanced pulmonary fibrosis.

(Internal Medicine 41: 1118-1123, 2002)
\end{abstract}

Key words: idiopathic pulmonary fibrosis, usual interstitial pneumonia, interstitial pneumonia associated with systemic sclerosis, therapeutic effects, adverse effects

\section{Introduction}

A reevaluation of IIP subgroups clearly confirms that patients with idiopathic pulmonary fibrosis/usual interstitial pneumonia (IPF/UIP) have an unfavorable prognosis with a median survival of 2-3 years (1-7). The current consensus is that IPF/ UIP is a progressive illness for which routine therapies are minimally effective $(8,9)$. Though nonspecific interstitial pneumonia (NSIP) lesions generally respond better to corticosteroids and have a favorable prognosis, a substantial number of NSIP patients with more fibrotic histologies have shown rather poor prognoses $(3,10)$. In addition to the IIP cases, either UIP or fibrotic NSIP histology can be found in the majority of patients with interstitial pneumonia associated with diffuse systemic sclerosis (IP-SSc) (11-13).

Corticosteroids and immunosuppressants have been the mainstay of therapy in chronic pulmonary fibrotic disorders such as IPF, NSIP and IP with SSc. Many studies have reported that $10 \%$ to $30 \%$ of patients with IPF show an initial, objective improvement with this form of therapy $(8,9)$. However, as the clinical courses are chronic, it is difficult to predict a longterm prognosis based on a short-term therapeutic effect. No feasible interventions have yet been found to prevent the disease from progressing to chronic respiratory failure, the main cause of death in patients with pulmonary fibrosis (14-16). However, several recent studies using new agents have yielded encouraging data. A combination of gamma-interferon with low-dose prednisolone brought about improvements in pulmonary function (17). Similarly, pirfenidone, a new antifibrotic agent that suppresses the production of inflammatory cytokines, was shown to prevent the deterioration of pulmonary fibrosis (18). In the latter study, the therapeutic effect of pirfenidone was seen even in advanced cases in whom both immunosuppressive and cytotoxic therapies had failed. In the present study, we evaluated the feasibility of this new anti-fibrotic agent in patients with IPF and IP-SSc whose pulmonary function had deteriorated and who required compassionate-use treatment.

From the Department of Respiratory Medicine, Graduate School of Medicine, Kyoto University, Kyoto and *Narita Memorial Hospital, **KDL Inc. Received for publication February 7, 2002; Accepted for publication September 6, 2002

Reprint requests should be addressed to Dr. Sonoko Nagai, the Department of Respiratory Medicine, Graduate School of Medicine, Kyoto University, 54 Kawahara-cho, Shogoin, Sakyo-ku, Kyoto 606-8507 


\section{Methods}

\section{Study Population (Table 1)}

Thirteen symptomatic, consenting patients under treatment at the Department of Respiratory Medicine at Kyoto University Hospital were enrolled in this open-label, compassionateuse study. We excluded patients whose conditions suggested the presence of any active infectious problem or malignancy requiring an immediate specific treatment.

At the time of treatment, from 1996 to 1998, pirfenidone (Marnac Inc., Dallas, TX) had to be imported by KDL Inc. (Tokyo) and with the permission of the Ministry of Health and Welfare of Japan. Data on 3 patients who suffered from pulmonary hypertension at the time of entry were not included in the follow-up study, as these patients died of cardiac failure secondary to the progression of pulmonary hypertension within 3 months after commencing the pirfenidone therapy. Among the 10 remaining patients included in the follow-up, 8 patients were diagnosed with IPF and 2 were diagnosed with IP-SSc. The interstitial pneumonia was diagnosed as UIP by surgical lung biopsy in 7 of the 8 patients with IPF and in one patient with IP-SSC. The other 2 patients were clinically diagnosed with IPF, based on CT findings characteristic of UIP (honeycombing distributed at subpleural areas and traction bronchiectasis with minimal ground glass opacities) and BAL fluid cell findings compatible with IPF/UIP (absence of lymphocytosis with a moderate increase in neutrophils) (2).

All 10 of the patients were males, and the median age was 65 years old (range, 46 to 70 years old). Two of the subjects were nonsmokers and 8 were ex-smokers. One patient had pulmonary infection (aspergilloma) at entry. Three patients were treated with orally administered prednisolone $(10 \mathrm{mg} / \mathrm{day})$ and 2 were treated with a combination therapy of prednisolone (10 $\mathrm{mg}$ /day) and azathioprine (50-100 mg/day). All patients were treated by domiciliary oxygen therapy at the time of entry. All patients participating in this study gave their informed consent, and the study was approved by the ethical committee of Kyoto University.

\section{Pirfenidone treatment}

The treatment protocol was planned according to Raghu's protocol $(18,19)$, with slight modifications based on Raghu's recommendations. Pirfenidone was administered orally to the patients after a 7-day serial examination of the time course of the plasma drug concentration. The initial loading dose was $400 \mathrm{mg}$ of pirfenidone given as a single dose. This was followed by single daily doses of $20 \mathrm{mg} / \mathrm{kg}$ body weight for three days, single daily doses of $30 \mathrm{mg} / \mathrm{kg}$ for four days, and divided doses (qid) of $40 \mathrm{mg} / \mathrm{kg}$ for one year. The plasma concentration of pirfenidone was monitored serially.If adverse effects such as photosensitivity appeared, the drug administration was temporarily withdrawn without tapering (for 14-28 days). At the completion of the treatment, the pirfenidone could be abruptly withdrawn without adverse effects.

\section{Measurement of the plasma concentration of pirfenidone}

The plasma concentration of pirfenidone was measured using high-performance liquid chromatography (HPLC) (20). The samples were preserved in frozen form $\left(-40^{\circ} \mathrm{C}\right)$ until use. At the time of measurement, the plasma were lysed at room temperature, and treated with OASIS TM HLB (Waters Co., Ltd., Milford, MA), for loading into HPLC.

The HPLC system consisted of a 600E Multisolvent Delivery System, 717 pulse Autosampler, 996 Photodiode Array Detector (310 nm), Scanning Fluorescence Detector (315 nm for activation and $375 \mathrm{~nm}$ for detection), and Symmetry C18 column $(4.6 \times 150 \mathrm{~mm})$ (Waters Co., Ltd.). Measurements were made under a column temperature of $35^{\circ} \mathrm{C}$ and flow rate of 1.5 $\mathrm{ml} / \mathrm{min}$, and a loading volume of $10 \mu \mathrm{l}$ methanol $20 \mathrm{mM}$ phos-

Table 1. Study Population at the Time of Treatment

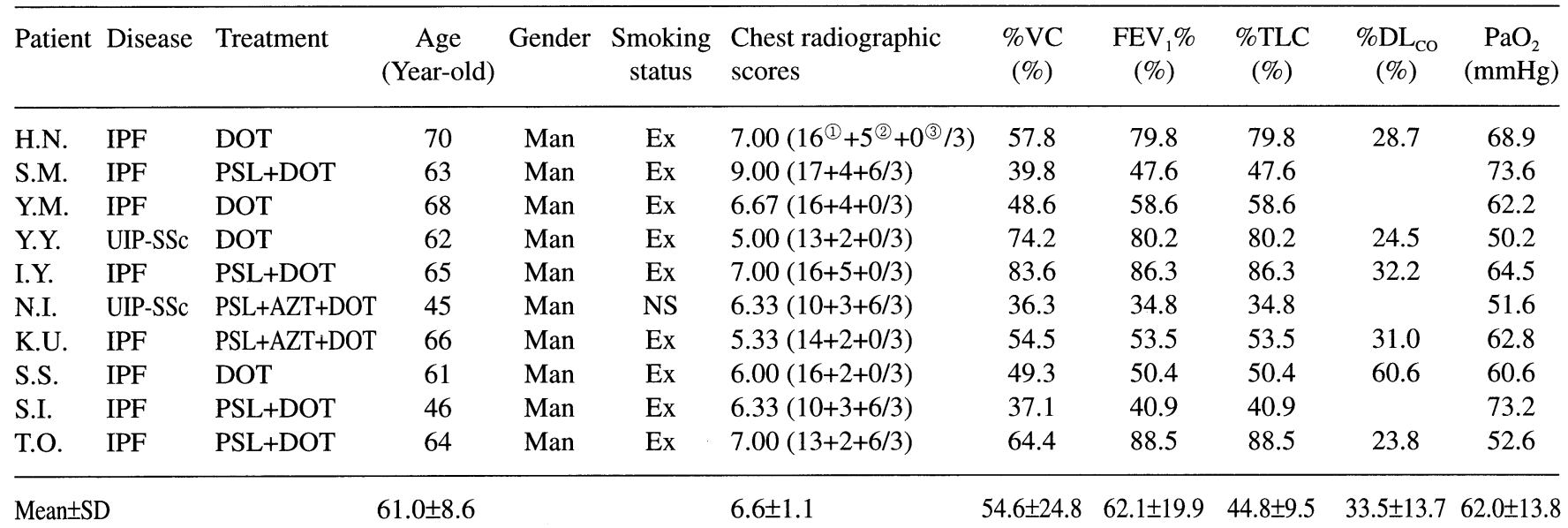

DOT: Domiciliary oxygen therapy, PSL: prednisolone, AZT: azathioprine, Ex: ex-smoker, NS: nonsmoker, Chest X-p scores measured according to Watter's method (21), $\mathrm{PaO}_{2}$, under supplemented oxygen $\left(1 \mathrm{l} / \mathrm{min}\right.$, nasal cannula) at rest. ${ }^{1}$ regional extent and severity/profusion of parenchymal interstitial infiltrates, ${ }^{2}$ radiographic honeycombing, ${ }^{3}$ radiographic evidence of pulmonary hypertension. 
phate buffer $(\mathrm{pH} 4.5): 50: 50 \mathrm{v} / \mathrm{v}$ was used in the extracting phase. Tetracaine-HCL (Wako, Tokyo) was used as an internal standard.

The plasma concentration of pirfenidone was serially measured (at $0,15,60,90$ minutes and 2, 4, 6, 8 and 24 hours) on the day of the initial loading and on day 8 after the initial loading. The time course of the plasma concentration of pirfenidone was analyzed using the one-compartment model method. T max (peak concentration of pirfenidone) and AUC (area under curve) were compared among the patients who took pirfenidone.

\section{Intervention and follow-up}

Medical history and physical examination data of the 10 patients were collected from our hospital medical records. In assessing pre-entry measurement, radiographic scores and pulmonary function tests were selected as indexes for evaluation.

Chest radiographic scores were measured according to the method of Watters et al (21). Briefly, we evaluated the standard posteroanterior chest radiographs using a quantitative score (maximum 10 points). The sum of the scores of 3 radiographic entities were included in the overall radiographic score: 1) regional extent and severity/profusion of parenchymal interstitial infiltrates, 2) radiographic honeycombing, and 3) radiographic evidence of pulmonary hypertension. The regional extent and severity/profusion of interstitial infiltrates were considered collectively in order to calculate a parenchymal subscore. The regional extent included 6 zones defined by imaginary lines drawn at one-third and at two-thirds of the distance from the apex of the lung to the dome of the diaphragm in bilateral hemothoraces. The severity/profusion of parenchymal involvement was assessed by the combined profusion score (small, rounded opacities and /or linear, irregular densities), based on 3 groupings of the UICC profusion grading system. Chest radiographs were taken at $135 \mathrm{kV}$ under the maximum inspired condition using the same equipment at Kyoto University Hospital (Shimadzu, Kyoto).

Pulmonary function tests included measurements of vital capacity, total lung capacity, diffusion lung capacity for carbon monoxide (these values are shown as percentages of the predicted values: \%VC, FEV1\%, \% TLC, \% $\mathrm{DL}_{\mathrm{CO}}$, respectively), forced expiratory volume in one second (FEV1)/forced vital capacity (FVC)(\%), and pulmonary arterial oxygen pressure $\left(\mathrm{PaO}_{2}\right)$ (at rest under similar conditions: supplemented oxygen $1.0 \mathrm{l} / \mathrm{min}$. via nasal canula). $\mathrm{DL}_{\mathrm{CO}}$ (single breath) values were corrected for hemoglobin but not for alveolar gas volume. The measurement was performed using Chestag-55V (Chest Co. Ltd., Tokyo).

All patients enrolled in this study were followed at the Kyoto University Hospital.

\section{Evaluation of the therapeutic effects of pirfenidone on vari- ous clinical indexes}

First, we examined the therapeutic effects of pirfenidone on overall survival at two years after the one-year treatment. Next, we calculated measurable changes in chest radiographic scores, pulmonary function (\%VC, FEV1/FVC, \% TLC, $\left.\% \mathrm{DL}_{\mathrm{CO}}\right)$, and
$\mathrm{PaO}_{2}$. For comparison between the indexes one year prior to entry (therapy) and one year after entry, we calculated the changes in the radiographic scores, \%VC, FEV1\%, \% TLC, $\% \mathrm{DL}_{\mathrm{CO}}$, and $\mathrm{PaO}_{2}$ at rest while receiving the same fraction of supplemented oxygen. Specifically, the changes were expressed according to either of two formulae: 1) the percentages to the initial value ([value at the time of entry]-[value at one-year prior to entry]/[value at one-year prior to entry] $\times 100(\%))$; or 2) ([value at one-year after entry]-[value at the time of entry]/ [value at the time of entry] $\times 100(\%))$. The two calculated percentages [(1), (2)] were compared using the paired Student's ttest in order to determine differences between pre-entry and post-entry.

Changes in symptoms were not considered in the evaluation of therapeutic effects, as the patients' hopes for effective treatment were thought to introduce a possible emotional bias. Data on the subjects whose treatment was temporarily interrupted (from 2 weeks to one month) due to the appearance of photosensitivity were included in the analysis.

\section{Statistical analysis}

Data were evaluated on a Macintosh personal computer using JMP software (SAS Institute Inc, Cary, NC) (22). Numerical data and measurable changes were analyzed using the Median test, and pairs were compared using the Student's t-test (23). Probability values of less than 0.05 were considered to indicate statistical significance.

\section{Results}

\section{Serial changes in disease severity}

The serial decline of pulmonary functions and chest radiographic scores over a two-year period (from one year prior to one year after entry) are shown in Table 2. During pirfenidone treatment both the reduction in $\mathrm{PaO}_{2}$ (Fig. 1 and Table 3) and the deteriorating chest radiographic scores (Fig. 2 and Table 3) seemed to be stabilized. The other indexes (\%VC, FEV1/FVC, $\% \mathrm{TLC}$, and $\% \mathrm{DL}_{\mathrm{CO}}$ ) were unchanged between pre- and posttreatment.

Table 2. Disease Severity

\begin{tabular}{lccc}
\hline Indexes & \multicolumn{3}{c}{ Time of examination } \\
\hline & Pre-Tr. & at the entry & Post-Tr. \\
\hline Chest X-p scores & $5.23 \pm 1.02 *$ & $6.56 \pm 1.1$ & $6.90 \pm 1.20$ \\
\hline$\% \mathrm{VC}(\%)$ & $56.7 \pm 15.2$ & $54.6 \pm 15.8$ & $53.5 \pm 19.2$ \\
$\mathrm{FEV}_{1}(\%)$ & $68.6 \pm 18.4$ & $62.1 \pm 19.9$ & $59.3 \pm 25.9$ \\
$\% \mathrm{TCL}(\%)$ & $47.5 \pm 6.9$ & $44.8 \pm 9.5$ & $46.5 \pm 15.1$ \\
$\% \mathrm{DL}_{\mathrm{CO}}(\%)$ & $34.7 \pm 6.1$ & $33.5 \pm 13.7$ & $30.2 \pm 10.9$ \\
$\mathrm{PaO}_{2}(\mathrm{mmHg}) * *$ & $77.6 \pm 10.5$ & $62.0 \pm 8.5$ & $62.9 \pm 10.9$ \\
\hline
\end{tabular}

*mean \pm SD. $* *$ measured under supplemented oxygen $(1 \mathrm{l} / \mathrm{min}$ nasal cannula) at rest. Pre-Tr.: One year prior to the beginning of treatment, Post-Tr.: One year after the begining of treatment. 

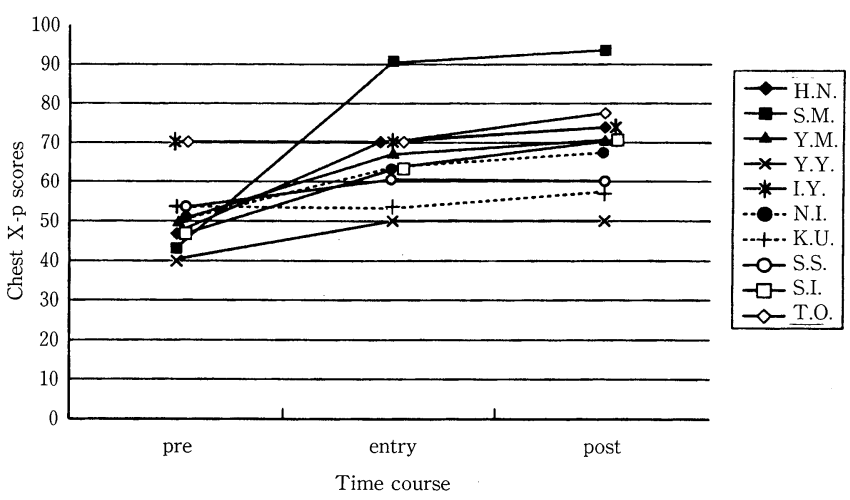

Figure 1. Serial changes in chest radiographic scores during the study period. The abscissa expresses the time of chest radiographs, and the ordinate expresses chest radiographic scores measured using Watter's method (21). Dotted lines (Fig. 1-3): patients who were treated with prednisolone and azathioprine.

Table 3. Therapeutic Effects of Pirfenidone

\begin{tabular}{lccc}
\hline Indexes & $\begin{array}{c}\text { (1) Pre-treatment } \\
\text { (median) } \\
\text { (min-max) }\end{array}$ & $\begin{array}{c}\text { (2) Posr-treatment } \\
\text { (median) } \\
\text { (min-max) }\end{array}$ & p-value \\
\hline Chest X-p score & 25.8 & 4.8 & 0.05 \\
& $(0 \sim 39.1)$ & $(2.8 \sim 7.2)$ & \\
$\% \mathrm{VC}(\%)$ & -3.15 & -2.55 & $\mathrm{NS}$ \\
& $(-17.5 \sim 9.7)$ & $(-13.5 \sim 12)$ & $\mathrm{NS}$ \\
$\mathrm{FEV}_{1}(\%)$ & -3.95 & -3.5 & \\
& $(-13.0 \sim-0.23)$ & $(-14.1 \sim 3.9)$ & $\mathrm{NS}$ \\
$\% \mathrm{TLC}(\%)$ & -4.1 & -1 & \\
& $(-6.8 \sim 6.5)$ & $(-9.7 \sim 18.1)$ & $\mathrm{NS}$ \\
$\% \mathrm{DL}_{\mathrm{CO}}(\%)$ & -6.6 & 0.4 & $(-3.5 \sim 22.9)$ \\
$\mathrm{PaO}_{2}(\mathrm{mmHg})$ & $(-2.5 \sim 23.9)$ & 1.95 & 0.05 \\
& -15.2 & $(-8.1 \sim 12.3)$ & \\
\hline
\end{tabular}

Formula to calculate values: (1)the percentages to the initial (([value at the time of entry]-[value at one-year prior to entry])/[value at oneyear prior to entry] $\times 100(\%) ;(2)$ the percentages to the values at the time of entry (([value at one-year after entry]-[value at the time of entry] $) \times 100(\%))$. The two calculated percentages $((1),(2))$ were compared using the paired Student's t-test in order to determine differences between pre-entry and post-entry.

\section{Plasma concentration of pirfenidone}

Based on the time profile, the plasma pirfenidone concentration as measured serially following a single oral administration $(400 \mathrm{mg}$ ) is shown in Fig. 3, the plasma concentration varied $(2.95-7.19 \mu \mathrm{g} / \mathrm{ml}$ in Cmax, $16.86-66.38 \mu \mathrm{g} \bullet \mathrm{h} / \mathrm{ml}$ in AUC $0-24$ ) among the 10 patients, and no correlations were found between dose and occurrence of either adverse or therapeutic effects within this dose range. The plasma concentration during the one-year treatment was well recorded though the range differed among patients (Table 4).

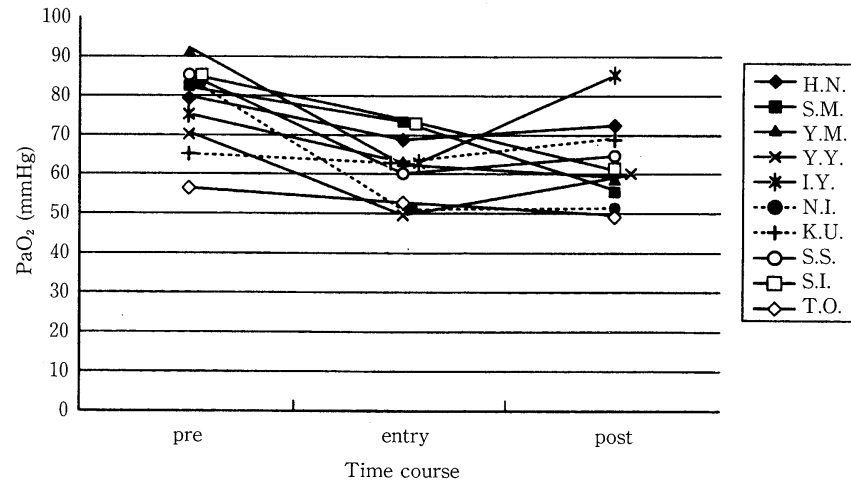

Figure 2. Serial changes in $\mathrm{PaO}_{2}(\mathrm{mmHg})$ during the study period. The abscissa expresses the time of arterial blood sampling, and the ordinate expresses $\mathrm{PaO}_{2}(\mathrm{mmHg})$ at rest under supplemeted oxygen $(1.0 \mathrm{l} / \mathrm{min}$. via nasal cannula).

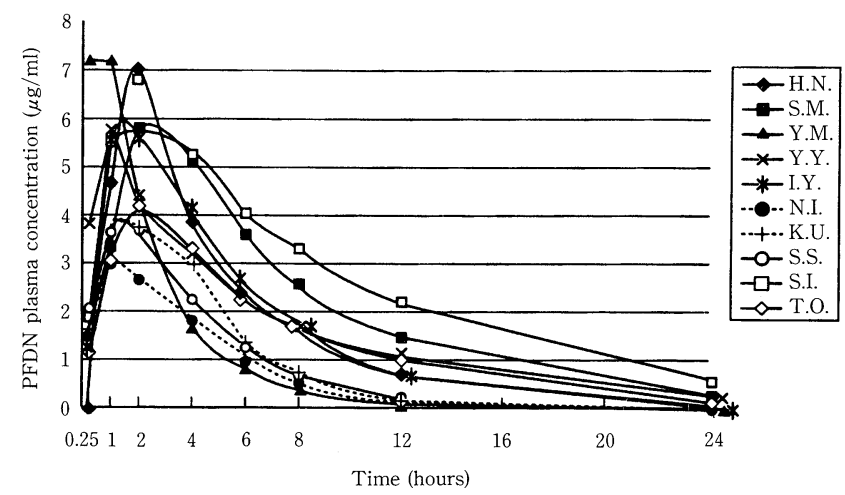

Figure 3. Plasma concentration of pirfenidone. The abscissa express the time (hours) of venous blood sampling after taking pirfenidone, and the ordinate expresses the plasma concentration of pirfenidone (microgram/ml).

\section{Clinical courses after treatment and cause of death}

The 10 patients were followed for 2 years after the one-year treatment with pirfenidone. The disease progressed with or without pulmonary hypertension in 8 patients, and remained stable in 2 patients. Among the 10 treated patients, 1 died of lung cancer in association with progression of pulmonary fibrosis within 2 years after treatment, and 3 died of cardiac failure due to pulmonary hypertension in association with progression of pulmonary fibrosis within 2 years after treatment. The remaining 2 patients died of respiratory failure due to disease progression within 2 years after treatment.

\section{Complications and adverse effects during pirfenidone treat- ment}

Four episodes of bronchopneumonia were recorded during the one-year treatment, but all cases were treated effectively with antibiotics. Three patients developed symptoms of photo- 


\section{NAGAI et al}

Table 4. Plasma Concentration of Pirfenidone during the One-year Treatment

\begin{tabular}{|c|c|c|c|c|c|c|c|c|c|c|}
\hline \multirow[b]{2}{*}{ Case } & & \multicolumn{5}{|c|}{ Months after Treatment } & \multirow[b]{2}{*}{$\min }$. & \multirow[b]{2}{*}{$\max$. } & \multirow[b]{2}{*}{ CAM (mg/day) } & \multirow[b]{2}{*}{ Itraconazole (mg/day) } \\
\hline & & 1 & 3 & 6 & 9 & 12 & & & & \\
\hline 1 & H.N. & 17.3 & 10.2 & - & $10.6^{*}$ & $4.0^{*}$ & 4.0 & 17.3 & 200 & 200 \\
\hline 2 & S.M. & 8.4 & 7.6 & - & $4.9 *$ & $4.9^{*}$ & 4.9 & 8.4 & - & - \\
\hline 3 & Y.M. & 2.3 & 2.4 & 2.4 & - & 2.7 & 2.3 & 2.7 & - & - \\
\hline 4 & Y.Y. & 10.4 & 8.0 & $4.9^{*}$ & $5.2 *$ & $6.0^{*}$ & 4.9 & 10.4 & - & - \\
\hline 5 & I.Y. & 3.4 & 6.9 & - & $4.0^{*}$ & $6.6^{*}$ & 3.4 & 6.9 & - & 200 \\
\hline 6 & N.I. & 5.1 & 4.4 & - & 3.1 & 3.8 & 3.1 & 5.1 & - & - \\
\hline 7 & K.U. & 13.3 & 19.7 & 6.8 & $15.2^{*}$ & - & 6.8 & 19.7 & 200 & - \\
\hline 8 & S.S. & 6.9 & 6.12 & 10.9 & - & 4.6 & 4.6 & 10.9 & - & - \\
\hline 9 & S.I. & 5.2 & 7.0 & 3.2 & 4.0 & 3.4 & 3.2 & 7.0 & - & - \\
\hline 10 & T.O. & 10.1 & - & 5.9 & - & - & 5.9 & 10.1 & - & - \\
\hline
\end{tabular}

pirfenidone $\mu \mathrm{g} / \mathrm{ml}$ at 4 hours after taking the drug (40 mg/kg b.w), $*(30 \mathrm{mg} / \mathrm{kg} \mathrm{b.w})$, CAM (clarithromycin) and/or Itraconazole: administered simultaneously (p.o.).

sensitivity ( 2 cases within the first 3 months of treatment, 1 case within the first 5 months of treatment) but recovered promptly when the drug was temporarily suspended. Five patients showed signs of anorexia within 1 month, and 2 patients complained of malaise or somnolence within 3 months. However, no liver dysfunction, hematological abnormalities, or allergic or shock reactions were seen during treatment. No withdrawal syndrome was appeared after suspending pirfenidone.

\section{Discussion}

In this open-label study, we demonstrated that there was no significant deterioration in chest radiographic score or pulmonary function data during the one-year treatment with pirfenidone and that no significant adverse effects occurred during or after suspending treatment in 10 patients with chronic pulmonary fibrosis, including IPF/UIP and IP-SSc.

We could not measure serial changes in CT scores, as some of the patients enrolled in this study had no CT data one-year prior to treatment with pirfenidone. However, we can report that the majority of patients showed a progression of fibrosis from small-sized honeycombing to enlarged cystic changes 2 years after the one-year treatment with pirfenidone.

These effects did not prolong the overall survival in the treated patients. This may have been due to the relatively short therapeutic duration of one year. We restricted the duration of pirfenidone therapy to one year because the permissible dosage of this drug for long-term therapy was restricted at the time of this study (1996) in Japan. This report is the second to examine therapeutic aspects of pirfenidone for patients with chronic pulmonary fibrosis. Additional large-scale, doubleblinded prospective studies will be needed to confirm the therapeutic effect of pirfenidone in patients with pulmonary fibrosis, particularly those with UIP-type fibrosis or fibrotic NSIP lesions, conditions that have particularly unfavorable prognoses $(3,9)$. In the first open-label study, Raghu and coworkers (18) compared the results at entry with the results after 2 years of treatment, while we compared the rates of deterioration at 1 year prior to treatment with those after 1 year of treatment.

Honeycombing on chest $\mathrm{CT}$ with traction bronchiectasis was found in all cases, and no acute episodes suggestive of superimposed infections, drug toxicity, or malignancy were detected during the treatment period. Therefore, the outcome of this study as an evaluation of the therapeutic effects of pirfenidone in patients with chronic pulmonary fibrosis (IPF/UIP and UIP$\mathrm{SSc}$ ) may be reliable.

Regarding the adverse effects, the appearance of skin photosensitivity required a one-month cessation of treatment in two cases, but the treatment could be reinitiated in both of these patients. No trouble emerged when the drug was abruptly withdrawn, either midway through the therapy or after its completion.

The plasma concentration of pirfenidone did not seem to relate to the occurrence of adverse effects, or to differences in therapeutic effects within the present dose range. Although the pharmacokinetic mechanisms of pirfenidone remain to be clarified, the present information on the plasma pirfenidone concentration should be applicable to future estudies on the drug's metabolism and anti-fibrotic mechanism.

In conclusion, we confirmed the feasibility of pirfenidone as an anti-fibrotic therapeutic drug in 10 patients with chronic pulmonary fibrosis at varying degrees of disease deterioration.

Acknowledgements: We greatly appreciate the generosity of Dr. Solomon B. Margolin, MS, PhD, and Nancy A. Cox, MD, PhD, (Marnac Inc., Dallas, TX) for providing pirfenidone for this study. We also thank our young staff for their support of the patients in the ward, and Mr. Simon Johnson for his help with the wording of this manuscript. This study was supported by Grants-inAid for Scientific Research from the Ministry of Science and Education of Japan (Nos. 10670540 and 12670557). 


\section{Pirfenidone Therapy to Lung Fibrosis}

\section{References}

1) Katzenstein AL, Myers JL. Idiopathic pulmonary fibrosis: Clinical relevance of pathologic classification. Am J Respir Crit Care Med 157: 1301$1315,1998$.

2) Nagai S, M. Kitaichi, T. Izumi. Classification and recent advances in idiopathic interstitial pneumonia. Curr Opin in Pul Med 4: 256-260, 1998.

3) Travis WD, Matsui K, Moss J, Ferrans VJ. Idiopathic nonspecific interstitial pneumonia: Prognostic significance of cellular and fibrosing patterns. Survival comparison with usual interstitial pneumonia and desquamative interstitial pneumonia. Am J Surg Pathol 24: 19-33, 2000.

4) Bjoraker JA, Ryu JH, Edwin MK, et al. Prognostic significance of histopathologic subsets in idiopathic pulmonary fibrosis. Am J Respir Crit Care Med 157: 199-203, 1998.

5) Nagai $S$, Kitaichi M, Nishimura K, Itoh H, Izumi T, Colby TV. Idiopathic Nonspecific Interstitial Pneumonia/Fibrosis: Comparison with Idiopathic Pulmonary Fibrosis and Bronchiolitis Obliterans Organizing Pneumonia. Eur Respir J 12: 1010-1019, 1998.

6) Daniil ZD, Gilchrist FC, Nicholson AG, et al. A histologic pattern of nonspecific interstitial pneumonia is associated with a better prognosis than usual interstitial pneumonia in patients with cryptogenic fibrosing alveolitis. Am J Respir Crit Care Med 160: 899-905, 1999.

7) American Thoracic Society, European Respiratory Society. ATS/ERS international multidisciplinary consensus classification of idiopathic interstitial pneumonia. Am J Respir Crit Care Med 165: 277-304, 2002.

8) Hunninghake GW, Kalica AR. Approaches to the treatment of pulmonary fibrosis. Am J Respir Crit Care Med 151: 915-918, 1995.

9) King Jr. TE, Costabel U, Cordier JF, et al. Idiopathic pulmonary fibrosis: Diagnosis and treatment. International Consensus Statement. Am J Respir Crit Care Med 161: 646-664, 2000.

10) Nicholson AG, Colby TV, du Bois RM, Hansell DM, Wells A. The prognostic significance of the histopathologic pattern of interstitial pneumonia in patients presenting with the clinical entity of cryptogenic fibrosing alveolitis. Am J Respir Crit Care Med 162: 2213-2217, 2000.

11) Katzenstein ALA. Systemic diseases involving the lung. in: Katzenstein and Askin's Surgical Pathology of Non-Neoplastic Lung Disease. 3rd Ed.,
WB Saunders, Philadelphia, 1997: 168-192.

12) Silver RM. Interstitial lung diseases of systemic sclerosis. Int Rev Immunol 12: 281-291, 1995.

13) Wells AU, Cullinan P, Hansell DM, et al. Fibrosing alveolitis associated with systemic sclerosis has a better prognosis than lone cryptogenic fibrosing alveolitis. Am J Respir Crit Care Med 149: 1583-1590, 1994.

14) Mason RJ, Schwarz MI, Hunninghake GW, Musson RA. Pharmacological therapy for idiopathic pulmonary fibrosis: Past, present, and future. Am J Respir Crit Care Med 160: 1771-1777, 1999.

15) Raghu G, Depaso WJ, Cain K, et al. Azathioprine combined with prednisone in the treatment of idiopathic pulmonary fibrosis: A prospective double-blind, randomized, placebo-controlled clinical trial. Am Rev Respir Dis 144: 291-296, 1991.

16) Lynch JP, McCune WJ. Immunosuppressive and cytotoxic pharmacotherapy for pulmonary disorders. Am J Respir Crit Care Med 155: 395420, 1997.

17) Ziesche R, Hofbauer E, Wittmann K, Petkov V, Block LH. A preliminary study of long-term treatment with interferon gamma- $1 \mathrm{~b}$ and low-dose prednisolone in patients with idiopathic pulmonary fibrosis. N Eng J Med 341: 1264-1269, 1999.

18) Raghu G, Johnson WC, Lockhart D, Mageto Y. Treatment of idiopathic pulmonary fibrosis with a new antifibrotic agent, pirfenidone. Results of a prospective, open-label phase II study. Am J Respir Crit Care Med 159: 1061-1069, 1999.

19) Raghu G. Idiopathic pulmonary fibrosis: A need for treatment with drugs other than corticosteroids-a role for antifibrotic agents? Mayo Clin Proc 72: 285-287, 1997.

20) Taniyama M, Ohbayashi S, Narita M, et al. Pharmacokinetics of antifibrotic agent, pirfenidone, in haemodialysis patients. Eur J Clin Pharmacol 52: 77-78, 1997.

21) Watters LC, Talmadge EK, Schwarz MI, Waldron JA, Stanford RE, Cherniack RM. A clinical, radiographic, and physiologic scoring system for the longitudinal assessment of patients with idiopathic pulmonary fibrosis. Am Rev Respir Dis 133: 97-103, 1986.

22) JMP statistical software for the Macintosh. Carry NC., SAS Institute, 1994.

23) Dawson-Saunders B, Trapp RG. Basic and clinical biostatitstics. 2nd ed. Prentice-Hall International, London, 1994: 189-209. 